\title{
Foreword
}

\section{Social Disparities in Thoracic Surgery: Actionable Items}

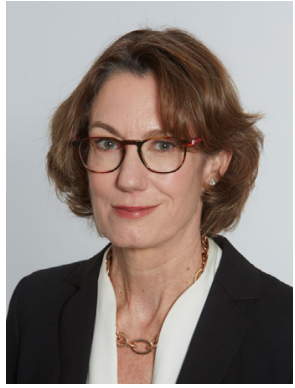

Virginia R. Litle, MD

Consulting Editor

We are excited to bring you this focused issue for the Thoracic Surgery Clinics on "Social Disparities in Thoracic Surgery." An established expert on this topic, Dr Cherie Erkmen, our guest editor, created a primer for how to address disparities not only in the management of lung cancer screening (LCS) but also in designing health disparities research projects, diversifying our thoracic surgery workforce, and working to level the playing field in health care delivery. Thanks to the novel coronavirus pandemic, these have become timely subjects, and the energy to make a difference is palpable not only in our clinical arena but also in our daily extracurricular life. Every day on social media (\#SoMe) and in the press we read about examples of social and health care disparities from police brutality to inequity in vaccine distribution. As surgeons, we are integral components of society. Our mission is to care for people. But also, as surgeons we like action and are accustomed to using a rulebook to accomplish our goals. The content of this issue provides guidance with actionable items. Example: how do you address disparities in LCS or esophagectomy rates? You educate primary care physicians about the LCS guidelines. You establish a centralized LCS program to unburden the workload of primary care physicians. You acknowledge that implicit bias has affected race- related differences in the patient-physician relationship, and then you provide unconscious bias training to your team so they can deliberately change. How do we optimize health care delivery? You can run for office and write your US senators and members of Congress; however, a more grass-roots approach includes supporting access to telehealth through your system and connecting with community leaders to address cultural challenges and the distrust in the health care system. Thank you to our outstanding contributors and thanks to the earnest effort of Dr Erkmen for providing a thorough compendium of socially relevant content for thoracic surgeons. Think globally and act locally.

Sincerely,

Virginia R. Litle, MD Section of Thoracic Surgery Cardiovascular Surgery Intermountain Healthcare

5169 So. Cottonwood Street, Suite 640 Murray, UT84107, USA

E-mail address: virginia.litle@imail.org Twitter: @vlitlemd (V.R. Litle) 